Research Article

\title{
A Study on Traditional Beliefs and Practices during the Postpartum Period among Mothers at a District Hospital in Goa
}

\author{
Preksha P Vernekar', Jagadish A Cacodcar ${ }^{2}$, Minaxi Panandikar ${ }^{3}$, lra Almeida ${ }^{4}$
}

${ }^{1}$ Assistant Lecturer, ${ }^{2}$ Professor and Head, Department of Community Medicine, Goa Medical College, Goa, India. ${ }^{3}$ Senior Gynaecologist, ${ }^{4}$ Senior Paediatrician, Directorate of Health Services, Goa, India.

DOI: https://doi.org/10.24321/2454.325X.202111

I $\quad \mathbf{N} \quad \mathbf{F} \quad \mathbf{O}$

\section{Corresponding Author:}

Preksha P Vernekar, Department of Community Medicine, Goa Medical College, Goa, India.

E-mail Id:

preksha.vernekar23@gmail.com

Orcid Id:

https://orcid.org/0000-0001-6008-024X

How to cite this article:

Vernekar PP, Cacodcar JA, Panandikar M, Almeida I. A Study on Traditional Beliefs and Practices during the Postpartum Period among Mothers at a District Hospital in Goa. Int J Preven Curat Comm Med. 2021;7(3):1-11.

Date of Submission: 2021-08-21

Date of Acceptance: 2021-09-21
$\begin{array}{llllllll}\mathbf{A} & \mathbf{B} & \mathbf{S} & \mathbf{T} & \mathbf{R} & \mathbf{A} & \mathbf{C} & \mathbf{T}\end{array}$

Background: Several mothers do not adhere to the standard medical advice given by health professionals as observed during medical practice and tend to follow potential harmful practices during the postnatal period. Little evidence is available in the state of Goa on the various traditional practices observed by mothers post-delivery.

Aim: To identify and explore various traditional beliefs and practices concerning newborn care and postpartum period among postnatal mothers at South Goa District Hospital.

Materials and Method: This descriptive, cross-sectional study was conducted over a period of 3 months using convenient sampling among admitted postnatal mothers. Data were entered into an Excel spreadsheet and analysed using SPSS version 22 .

Results: Majority of the study participants breastfed their newborn babies within 24 hours of childbirth (97.50\%). 96.25\% of the mothers fed colostrum to their newborn babies. A significant proportion of mothers used new washed clothes for their babies (83.75\%) and bathed them five days post-delivery (72.5\%). No dressing was applied to baby's umbilical stump (58.75\%). Postpartum home confinement with newborn baby for 40 days was observed by $22.5 \%$ of the mothers. Mothers applied kajal to the baby's face and eyes (50\%), used bangles (35\%) and anklets (16.25\%) to protect baby from evil eye.

Conclusion: The study highlighted the need for awareness of health professionals about the popular traditional practices observed among postnatal mothers in order to eliminate possible harmful practices and reinforce beneficial practices for a healthy postpartum period for the mother.

Keywords: Cultural, Practices, Beliefs, Postnatal, Mothers 


\section{Introduction}

The postpartum period is an important transition time for a woman to recuperate after pregnancy and childbirth and to take on a new role as a mother. This precarious phase, especially the first six weeks, also renders the new mother vulnerable to illness, and specific traditional practices are observed to ensure recovery and avoid ill health in later years. ${ }^{1}$

In India, certain practices relating to pregnancy, childbirth and child development have stemmed from cultural beliefs, customs, and traditions that are based on that knowledge contained in ancient Indian texts. ${ }^{2}$ These cultural practices exist in both rural and urban Indian communities with varied differences. Many women in India follow these specific practices during their pregnancy, childbirth, and postnatal period. These specific practices are deemed important owing to the value attached to orally disseminated knowledge by elders.

While childbirth is a biological event, the experience of motherhood is mostly a social construct, shaped by cultural perceptions and practices. Besides these, other factors such as a woman's age, literacy, socioeconomic status, ethnic background, religion, and culture have a significant influence on child-rearing. Previously published literature from across India have reported harmful postpartum practices, some of which include delayed initiation of breastfeeding, giving pre-lacteal feeds, restriction of nutritious food and water consumption, early bathing of newborns, and unhygienic practices relating to cord care and personal hygiene. ${ }^{3}$ While some customs and beliefs are known to be harmful, some of them may have beneficial effects while others may be harmless or useless to maternal and infant health.

Researchers have argued that the medical view of pregnancy and birth often fails to appreciate the influence that traditional beliefs and practices have on the utilisation of maternal healthcare services. Over the last decade, public health approaches to maternal and newborn health in India have emphasised the need for access to health care services, chiefly skilled attendance, and emergency obstetric care, but have paid comparatively less attention to improving family and community practices and to the significance of cultural factors in health and illness. ${ }^{4}$ This lack of sensitivity towards traditional customs and beliefs can possibly lead to an inefficient doctor-patient relationship. Provision of culturally competent obstetric health care demands planning and implementation of high-quality health services that are sensitive to the diverse cultural background of the mothers.

It has been noted that there is a paucity of data on traditional beliefs and cultural practices related to pregnancy and childbirth from Goa. Therefore, this study was undertaken to gather insight on traditional household practices among postnatal mothers admitted at South Goa District Hospital to make suitable recommendations and suggest effective strategies for the promotion of healthy newborn and postnatal care practices that require an understanding of the community and to promote a culturally sensitive and acceptable change in outlook towards postnatal care.

\section{Materials and Methods}

The present study was an observational descriptive hospital based study conducted in the in-patient department of Obstetrics \& Gynaecology at South Goa District Hospital over a period of 3 months (March-May 2019).

Mothers within the 72 hours postnatal period who deliverd either by vagina or lower segment caesarean section delivery and admitted in the respective wards of the Hospital were selected. Convenient sampling technique was used to select 80 postnatal mothers for data collection during the study period. Lactating mothers who had at least 1 living child less than 3 years of age were eligible to be part of the study. Primigravida and those who did not wish to give consent were excluded from the study. Data were collected thrice weekly throughout the study period.

The purpose of the study was explained before collecting the data and written informed consent was obtained from all the participants. A pre-designed, pre-tested, semistructured questionnaire was used to conduct a direct face to face interview with the study participants. The questionnaire was developed based on the objectives of the study and on previously published literature. It consisted of 3 parts as follows:

Part I: Socio-demographic data of the study participants like age, sex, religion, occupation, education, etc. (Modified B. G. Prasad Classification, Update - 2019 was used to classify participants based on their socio-economic status).

Part II: Newborn care beliefs and practices after childbirth.

Part III: Cultural beliefs and practices during the immediate postnatal period.

The study began after obtaining ethical approval from the Institutional Ethics Committee (IEC) of Goa Medical College.

Data were entered into Microsoft Excel spreadsheet and analysed using SPSS version 22. Descriptive statistics were used to present the data.

\section{Results}

The socio-demographic characteristics of the study participants as depicted in Table 1 showed that exactly half of the mothers were aged between 19 and 25 years, $53.75 \%$ of them were Hindus, and $58.75 \%$ of them resided in a rural area. Majority of the mothers were locals (61.25\%). More than half of the mothers belonged to nuclear families 
(52.5\%). A considerable portion of the study participants had received lower primary school education (22.5\%) while another $21.25 \%$ had received high school education. Although only $13.75 \%$ of the mothers were illiterate, $93.75 \%$ of them were unemployed. Almost half of the mothers (41.25\%) belonged to Class III socioeconomic status as per modified B. G. Prasad Classification, Update - 2019. Nearly two-thirds of the mothers, i.e., $70 \%$ had a single child.

Table I.Sociodemographic Characteristics of Study Participants

\begin{tabular}{|c|c|}
\hline & Nhumbor to \\
\hline Variables & Number (\%) \\
\hline \multicolumn{2}{|l|}{ Age (in years) } \\
\hline$\leq 18$ & $4(5.0)$ \\
\hline $19-25$ & $40(50.0)$ \\
\hline$>25$ & $36(45.0)$ \\
\hline \multicolumn{2}{|l|}{ Religion } \\
\hline Hindu & $43(53.75)$ \\
\hline Catholic & $12(15.0)$ \\
\hline Muslim & $25(31.25)$ \\
\hline \multicolumn{2}{|l|}{ Residence } \\
\hline Rural & $47(58.75)$ \\
\hline Urban & $33(41.25)$ \\
\hline \multicolumn{2}{|l|}{ Origin } \\
\hline Local & $49(61.25)$ \\
\hline Migrant & $31(38.75)$ \\
\hline \multicolumn{2}{|l|}{ Type of family } \\
\hline Nuclear & $42(52.5)$ \\
\hline Joint & $16(20.0)$ \\
\hline Three-generation & $22(27.5)$ \\
\hline \multicolumn{2}{|l|}{ No. of living children } \\
\hline 1 & $56(70.0)$ \\
\hline 2 & $18(22.5)$ \\
\hline 3 & $6(7.5)$ \\
\hline \multicolumn{2}{|l|}{ Education } \\
\hline Illiterate & $11(13.75)$ \\
\hline Lower primary & $18(22.5)$ \\
\hline Upper primary & $12(15.0)$ \\
\hline Middle school & $11(13.75)$ \\
\hline High school & $17(21.25)$ \\
\hline $\begin{array}{l}\text { Higher secondary/ Post } \\
\text { high school } \\
\text { diploma }\end{array}$ & $8(10.0)$ \\
\hline Graduate and above & $3(3.75)$ \\
\hline
\end{tabular}

\begin{tabular}{|c|c|}
\hline Occupation \\
\hline Unemployed & $75(93.75)$ \\
\hline Skilled worker & $4(5.0)$ \\
\hline Professional & $1(1.25)$ \\
\hline Socio-economic status \\
\hline Class I & $9(11.25)$ \\
\hline Class II & $21(26.25)$ \\
\hline Class III & $33(41.25)$ \\
\hline Class IV & $11(13.75)$ \\
\hline Class V & $6(7.5)$ \\
\hline
\end{tabular}

Table 2 shows the traditional practices observed by postnatal mothers with respect to breastfeeding. It was observed that a large majority of the study participants breastfed their newborn babies within 24 hours of childbirth (97.50\%), with $86.25 \%$ breastfeeding within 30 minutes after birth. Almost all the mothers fed colostrum to their newborn babies (96.25\%). In addition, none of these mothers gave or were advised to give pre-lacteal or formula feeds to their newborn babies.

As far as traditional practices related to the care of newborns among postnatal mothers are concerned, a significant proportion of mothers used new washed clothes for their newborns (83.75\%) and bathed them five days post-delivery (72.5\%). Majority of the study respondents did not apply any dressing to the umbilical stump of the baby (58.75\%). There was no desire for the burial of placenta in $92.5 \%$ of the mothers. With respect to the handling of the desiccated umbilical cord of the neonate, it was found that $42.5 \%$ of the mothers believed in burying the cord in the courtyard of the house, while $31.25 \%$ hid the cord in their homes (Table 3).

Table 4 highlights some selected traditional practices observed by postnatal mothers in the immediate postnatal period. Almost half of the study participants were advised to be confined along with the baby in the hospital till day 6 post-delivery $(48.75 \%)$. Nearly two-thirds of the mothers observed naming ceremony of the baby (72.5\%). Almost all babies were given massages before bath (98.75\%). Other traditional practices that were noted were exposing the baby to holy smoke (dhoop) after bath (30\%) and exposing the baby to warm sunlight to counteract jaundice (90\%).

Out of the 80 study participants, $22.5 \%$ observed a period of postpartum confinement of 40 days at home with their newborn babies.

As can be seen in Figure 1, some traditional practices adopted by postnatal mothers to protect baby from the evil eye were applying kajal to the baby's face (50\%), using bangles (35\%), tying black thread (11.25\%), and using anklets $(16.25 \%)$ or chains $(10 \%)$. 
Table 2.Traditional Practices related to Breastfeeding among Postnatal Mothers

$(\mathrm{N}=80)$

\begin{tabular}{|c|c|}
\hline Practices & Number (\%) \\
\hline \multicolumn{2}{|c|}{ Initiation of breastfeeding } \\
\hline Within 30 minutes of birth & $69(86.25)$ \\
\hline 30 minutes to 60 minutes after birth & $6(7.5)$ \\
\hline 1 hour to 24 hours after birth & $3(3.75)$ \\
\hline 24 hours to 48 hours after birth & $1(1.25)$ \\
\hline More than 48 hours after birth & $1(1.25)$ \\
\hline \multicolumn{2}{|c|}{ Feeding of colostrum to newborn baby } \\
\hline Yes & $77(96.25)$ \\
\hline No & $3(3.75)$ \\
\hline \multicolumn{2}{|c|}{ Giving pre-lacteal feeds to newborn baby } \\
\hline Yes & $0(0)$ \\
\hline No & $80(100)$ \\
\hline \multicolumn{2}{|c|}{ Giving formula feeds to newborn baby } \\
\hline Yes & $0(0)$ \\
\hline No & $80(100)$ \\
\hline
\end{tabular}

Table 3.Traditional Practices related to Care of Newborn among Postnatal Mothers

\begin{tabular}{|c|c|}
\hline Practices & Number (\%) \\
\hline \multicolumn{2}{|c|}{ Type of clothes used to wrap newborn baby } \\
\hline Old washed clothes & $13(16.25)$ \\
\hline New washed clothes & $67(83.75)$ \\
\hline \multicolumn{2}{|c|}{ Timing of first bath of newborn baby } \\
\hline Day 1 & $4(5.0)$ \\
\hline Day 2 & $6(7.5)$ \\
\hline Day 3 & $6(7.5)$ \\
\hline Day 4 & $6(7.5)$ \\
\hline Day 5 & $58(72.5)$ \\
\hline \multicolumn{2}{|c|}{ Application of dressing to umbilical stump } \\
\hline Oil & $6(7.5)$ \\
\hline Antiseptic & $27(33.75)$ \\
\hline None & $47(58.75)$ \\
\hline \multicolumn{2}{|c|}{ Desire for burial of placenta after childbirth } \\
\hline Yes & $6(7.5)$ \\
\hline No & $74(92.5)$ \\
\hline \multicolumn{2}{|c|}{ Handling of umbilical cord after it fell off } \\
\hline Burial & $34(42.5)$ \\
\hline Hid the cord in their home & $25(31.25)$ \\
\hline Kept cord in amulets & $9(11.25)$ \\
\hline None & $12(15.0)$ \\
\hline
\end{tabular}

Table 4.Selected Traditional Practices observed by Postnatal Mothers

$(\mathrm{N}=80)$

Practices Number (\%)

Confinement of mother in hospital till day 6 after delivery

\begin{tabular}{|c|c|}
\hline Yes & $39(48.75)$ \\
\hline No & $41(51.25)$ \\
\hline Naming ceremony of the baby \\
\hline Yes & $58(72.5)$ \\
\hline No & $22(27.5)$ \\
\hline Giving massage to baby before bath \\
\hline Yes & $79(98.75)$ \\
\hline No & $1(1.25)$ \\
\hline Exposing baby to dhoop after bath \\
\hline Yes & $24(30.0)$ \\
\hline No & $56(70.0)$ \\
\hline
\end{tabular}

Exposing baby to warm sunlight to decrease jaundice (if present)

\begin{tabular}{|c|c|}
\hline Yes & $72(90.0)$ \\
\hline No & 8 (10.0) \\
\hline \multicolumn{2}{|c|}{$\begin{array}{l}\text { Confinement of mother and baby at home for } 40 \\
\text { days postpartum }\end{array}$} \\
\hline Yes & 18 (22.5) \\
\hline No & 62 (77.5) \\
\hline
\end{tabular}

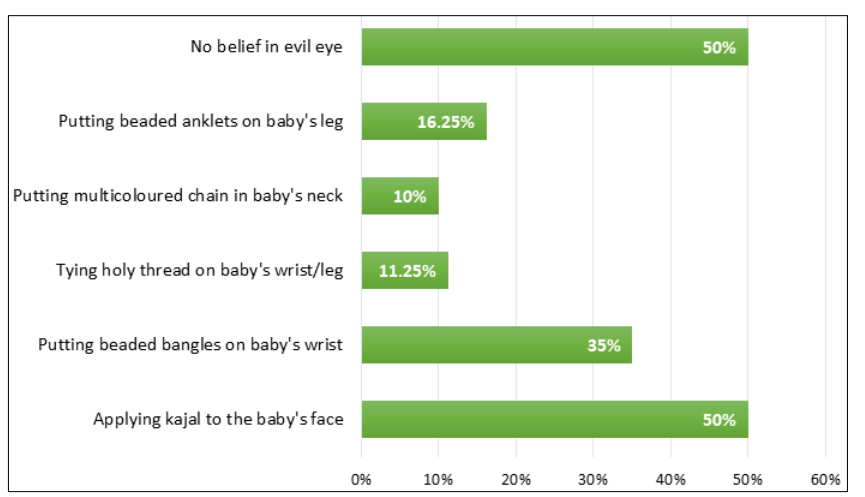

Figure I.Traditional Practices observed by Postnatal Mothers to Protect Baby from Evil Eye

\section{Discussion}

The present study highlights that a considerable amount of importance is given to cultural practices related to maternal and newborn care. These traditional practices observed in various parts of the country with changing frequencies have various levels of significance attached to them, owing to the transfer of these beliefs across generations as a component of socio-cultural hierarchy, despite advances 
in science and technology. A comprehensive review of evidence published in the past shows that mothers were subjected to harmful postpartum practices in spite of socio-economic development and educational competence. The possible emergence of these traditional practices is believed to have originated from the human instinct to protect and nurture life.

Our study showed positive attitudes towards exclusive breastfeeding among the study participants. Majority of women (86.25\%) initiated breastfeeding their newborn babies within 30 minutes of birth which was comparable to studies done in India and across countries. ${ }^{5,6,7}$ The mother's desire for early initiation of breastfeeding is considered a positive behaviour in terms of maternal and infant health. Breast milk fully meets the nutritional requirements of the infant in the first few months of life. It also boosts the mother's immune system and lowers the risk of postpartum haemorrhage and anaemia. ${ }^{8}$ Breastfeeding also facilitates rooming-in and promotes emotional bonding between the mother and baby in the early minutes after birth. Exclusive breastfeeding also leads to lactational amenorrhea which in turn acts as a form of natural contraception thereby allowing spacing between births. In addition, a high proportion of the study participants fed colostrum to their newborn babies $(96.25 \%)$ which was consistent with similar studies. ${ }^{9}$ In contrast, studies conducted in other regions of India observed that colostrum was believed to be a thick yellow non-nutrient liquid that causes harm and was difficult to digest for newborns. ${ }^{10}$ Colostrum contains antimicrobial factors such as secretory IgA, anti-streptococcal factor, lysozymes and lactoferrin which protect the newborn against diarrhoeal diseases and respiratory infections in the first few of life. ${ }^{8}$

From a medical perspective, artificial feeding in the form of breast milk substitutes is advised in cases of premature or low birth weight babies, failure to thrive and failure of production of breast milk, or chronic illness in the mother. In our study, none of the mothers gave or were advised to give pre-lacteal or formula feeds to their babies which probably is attributed to informed decisions taken by mothers secondary to receiving health education concerning breastfeeding practices. Breastfeeding is vital in the formation of healthy intestinal flora. Therefore, exposure to pre-lacteal or formula feeds foods as seen in the studies done by Nethra et al. in Karnataka ${ }^{11}$ and Memon et al. in Pakistan ${ }^{12}$ might lead to the breastfeeding practices becoming sub-optimal and may have an adverse impact on the developing digestive system of the babies. However, mothers residing in some rural regions of our country commonly believed that the child swallows waste and impurities in the womb, which need to be cleansed through pre-lacteal feeds such as honey, sugar or jaggery water, fresh butter/ghee or milk other than breast milk. ${ }^{13}$
As far as traditional newborn care practices are concerned, our study revealed a considerably favourable outlook towards the care of newborns. A large majority of postnatal women used new washed clothes to wrap newborn babies after birth (83.75\%) which was in contrast to qualitative studies done by Shah et al. in Eastern Nepal ${ }^{14}$ and Mersha et al. in Southern Ethiopia ${ }^{15}$ wherein mothers used old washed clothes to wrap newborn babies after delivery. The reason mentioned by the mothers for this probably beneficial practice of using old clothes which have also been used for previously born children in their families was the belief that such clothes tend to be softer and thinner in texture suitable for the delicate skin of their newborn child. An appreciable proportion of postnatal mothers in our study stated that their newborn child was bathed after 5 days post-delivery (72.5\%) which was distinct from a study conducted by Bahurupi et al. in Maharashtra ${ }^{16}$ wherein tribal women bathed their newborns within 24 hours post-delivery. Other studies done by Gedamu et al. ${ }^{17}$ in northwest Ethiopia and Chand SB in Nepal ${ }^{18}$ showed that mothers bathed their babies within 24 hours after delivery ( $45 \%$ and $31 \%$ respectively). The reasons for sub-optimal thermal care practices for newborn babies in these studies could be due to lack of awareness and associated sociocultural beliefs wherein newborn babies were considered as dirty or contaminated by mother's blood. ${ }^{15}$ Metabolic processes, including non-shivering thermogenesis, serve as the principal source of body heat in neonates. Newborns, especially those born preterm or those who are small for their gestational age are prone to excessive heat loss because they have a relatively large surface area in relation to body mass. Hence, practices such as bathing newborns within 24 hours of birth increase the risk of neonatal hypothermia with increased metabolic stress leading to complications such as hypoglycemia, hypoxia, and metabolic acidosis. ${ }^{19}$

With regard to beliefs surrounding the umbilical cord, our study established that $42.5 \%$ of the mothers buried it in their courtyard after it dried and fell off. This finding was congruent with similar studies conducted across India. ${ }^{11,20}$ A study performed by Karahan et al. ${ }^{21}$ observed that people harboured various beliefs with respect to burial of umbilical cord; in a school garden to ensure the child's education or in a mosque courtyard to rear a religious child, while some threw the cord remains into a nearby water body to ensure the child fulfils his/ her destiny elsewhere. The present study also showed that most of the study respondents did not apply any dressing to the cord stump (58.75\%) which was consistent with studies done by Gedamu et al. ${ }^{17}$ (54.4\%) and Mersha et al. ${ }^{14}$ (60.2\%). However, an exploratory study conducted in Uttarakhand by Saxena $V$ et al. ${ }^{22}$ noted the application of food substances such as oil, ghee, turmeric, and mother's milk to the umbilical stump 
of the baby. Harmful traditional cord care practices are one of the leading causes of neonatal sepsis in low and middleincome countries. ${ }^{23}$ The baby's umbilical stump is prone to bacterial infections owing to the underdeveloped immune system. The proclivity towards active umbilical cord care of a newborn could be utilised to inculcate positive behaviour change among the masses such as application of mild antiseptic to the cord stump and the skin around its base.

There was no desire for the burial of placenta in majority of the participants $(92.75 \%)$ in our study which was in contrast to the findings of a systematic review conducted by Dennis et al. ${ }^{1}$ that observed certain superstitious beliefs surrounding the burial of placenta across some regions of the world. This review highlighted that Malaysians believed that the placenta was the baby's 'sibling' and buried it after placing it in a 'winding' sheet and coconut shell, while among rural Koreans, the placenta was burnt on the third day postpartum and its ashes were buried to promote longevity of the child.

In addition to the above practices, certain traditional beliefs were assessed among the postnatal mothers after childbirth. Almost half of the study participants (48.75\%) were advised to confine in the hospital till day 6 post-delivery. This custom called Sotti or Shastipujan was observed mainly among Goan Hindu women who believe that on the night of the sixth day from the child's birth, the honouring of the female deity called Sottvai is done to the beat of the 'ghumott', a special local percussion instrument made from a mud-pot. ${ }^{24}$

In our study, it was also noted that $22.5 \%$ of the mothers were confined to their home with their newborn baby till 40 days postpartum as was evident in a study done by Morris et al. wherein a confinement period of 1 - 4 months was practised by $92 \%$ of postnatal women. ${ }^{25}$ In Indian societies, women are advised confinement for the first 40 days after birth to improve maternal well-being and nurture their newborn babies. From a medical perspective, the duration of the first 6 weeks after birth is considered a precarious phase for the development of complications such as bleeding and infection. ${ }^{21}$ However, it is believed that long periods of postpartum confinement may have a serious implication on the mental health of postnatal mothers aggravated by the health challenges during this period. It is desirable to decrease the duration of confinement to avoid postpartum depression and delay in seeking medical care. ${ }^{26}$

With respect to newborn babies, in the present study, the practice of giving oil massage to the baby daily before bath was reported by $98.75 \%$ of the mothers. This finding was consistent with studies done by Reshma et al. ${ }^{20}$ (95\%), Sasikala et al. ${ }^{27}(79.4 \%)$ and Bangari et al. ${ }^{28}(71 \%)$. Of the study participants, $72.5 \%$ indicated that they followed a special ritual when naming their children. Goan Hindu societies perform a ceremony locally called 'Barso' on the twelfth day after the child's birth. On this day, married women (excluding widows) meet and give gifts and flowers to the delivered woman by placing them in her lap. The child is placed in the cradle and is bestowed a name, by reciting it in the baby's ear before announcing it to the family. ${ }^{24}$ Catholics perform the sacrament Baptism to bless the child with a name. ${ }^{29}$ Muslims call the Pesh Imam or Bangi from a Masjid who says two prayers into the ears of the child; Azaan into the right ear and lkayat into the left. After this, honey is applied to the tongue of the child and it is bestowed a name. ${ }^{30}$

In the present study, 30\% of the study respondents exposed their newborn babies to camphor (Dhoop) after bath which is similar to a study conducted by Udgiri R in Vijayapura (34\%). ${ }^{31}$ This practice of exposure to holy smoke after bath should ideally be avoided as it may predispose the baby to respiratory infections, breathing difficulties or even allergies in later life.

With reference to the cultural practices surrounding care in case of jaundice in newborns, it was found that 72 (90\%) participants exposed their babies to warm sunlight which is consistent with the studies done by Sasikala et al. ${ }^{27}$ This practice is proven to help in decreasing the incidence of jaundice in children and is considered to be a healthy practice. However, the traditional practice of treating jaundice by branding of skin and using leaf extracts (pasaru) may cause more harm than good. Globally, neonatal hyperbilirubinemia is an important cause of neonatal morbidity and mortality and one of the most common reasons for hospital admission in the first week of life and neurodevelopmental impairment in developing countries. ${ }^{32}$ Safer options such as phototherapy are available at tertiary health care centres to counteract physiological jaundice.

Traditional practices intended to curb the wrath of the evil eye reflect the desire for a problem-free healthy growing child. In our study, strikingly, half of the study participants did not believe in seeking protection for their babies to ward off the evil eye. However, the other half applied kajal to their baby's face as seen by Sunanda et al. ${ }^{33}$ (99\%), used bangles (35\%) or black thread (11.25\%) as seen by Reshma et al. ${ }^{20}(66 \%)$, and anklets $(16.25 \%)$ or chains $(10 \%)$ made of multi-coloured beads. Bangari et al. ${ }^{28}$ also noted in their study that $57.3 \%$ of women believed that applying kajal to newborn babies on their eyes, face or behind the ear, is essential. However, mothers need to be educated regarding the consequences of application of kajal to the baby's eyes. Kajal (kohl) can cause itching or irritation in the eyes or can lead to infections such as blepharitis, conjunctivitis, or dacryocystitis. Fingernail trauma while applying kajal can cause conjunctival or corneal lacerations.

\section{Conclusion}

Cultural practices pertaining to newborn and postpartum 
care are deeply rooted in our societies as they are passed on from generation to generation. Our study showed favourable breastfeeding and newborn care practices such as early initiation and exclusive breastfeeding, delayed bathing of newborn and optimal cord care and also highlighted some good traditional customs and beliefs. While certain practices such as postpartum confinement are potential threats to sound physical and mental health of the mother and need to be stopped, some harmless practices such as naming ceremony of the child are reflective of celebration of the postpartum period and should be acknowledged to build a good rapport with mothers and their families. The health professionals should be aware of these traditional practices followed by the community to provide culturally appropriate and acceptable health care. It is recommended that harmless, acceptable or even potentially beneficial practices observed during the postnatal period should be supported and considered a part of our rich culture, whereas harmful practices should be avoided with health education provided at the time of discharge from hospital and during postnatal visits for health check-up of mother and baby.

\section{Source of Funding: None Conflict of Interest: None \\ References}

1. Dennis CL, Fung K, Grigoriadis S, Robinson GE, Romans $\mathrm{S}$, Ross L. Traditional postpartum practices and rituals: a qualitative systematic review. Womens Health (Lond). 2007 Jul;3(4):487-502. [PubMed] [Google Scholar]

2. Cousik R, Hickey MG. Pregnancy and childbirth practices among immigrant women from India: have a healthy baby. The Qualitative Report. 2016 Apr 18;21(4):72743. [Google Scholar]

3. Moola S, Tyagi J, Shah P, Nambiar D, Bhaumik S. Harmful postpartum beliefs and practices of mothers in India: rapid evidence synthesis. Vishakhapatnam: The George Institute for Global Health; Aug 2020. [Google Scholar]

4. Raman S, Srinivasan K, Kurpad A, Razee H, Ritchie J. "Nothing special, everything is maamuli": socio-cultural and family practices influencing the perinatal period in urban India. PLoS One. 2014 Nov 4;9(11):e111900. [PubMed] [Google Scholar]

5. Bala K, Sahni B, Bavoria S, Narangyal A. Knowledge, attitude, and breast-feeding practices of postnatal mothers in Jammu: A community hospital based cross sectional study. J Family Med Prim Care. 2020 Jul 30;9(7):3433-7. [PubMed] [Google Scholar]

6. Okka B, Durduran Y, Kodaz ND. Traditional practices of Konya women during pregnancy, birth, the postpartum period, and newborn care. Turk J Med Sci. 2016;46:50111. [PubMed] [Google Scholar]

7. Akhtaruzzaman M, Hossain MA, Karim MR, Khan RH,
Islam MS, Ahamed F, Khan N, Ahammed SU, Dhar SK, Mahmud AA, Khan AK, Nahar S. Attitude and practices of mothers on breastfeeding attended at a tertiary hospital in Bangladesh. Mymensingh Med J. 2015 Jul;24(3):480-5. [PubMed] [Google Scholar]

8. KE Elizabeth. Breastfeeding and BFHI. Nutrition and child development. 5th edition. Hyderabad, India: Paras Medical Publisher; 2015;2-29.

9. Sultania P, Agrawal NR, Rani A, Dharel D, Charles R, Dudani R. Breastfeeding knowledge and behavior among women visiting a tertiary care center in India: a cross-sectional survey. Ann Glob Health. 2019;85(1):64. [PubMed] [Google Scholar]

10. Bandyopadhyay M. Impact of ritual pollution on lactation and breastfeeding practices in rural West Bengal, India. Int Breastfeed J. 2009 Mar 26;4:2. [PubMed] [Google Scholar]

11. Nethra N, Udgiri R. A study on traditional beliefs and practices in newborn care among mothers in a tertiary health care centre in Vijayapura, North Karnataka. Int J Comm Med Public Health. 2018 Feb 24;5(3):1035. [Google Scholar]

12. Memon J, Holakouie-Naieni K, Majdzadeh R, Yekaninejad MS, Garmaroudi G, Raza O, Nematollahi S. Knowledge, attitude, and practice among mothers about newborn care in Sindh, Pakistan. BMC Pregnancy Childbirth. 2019 Sep 6;19:329. [PubMed] [Google Scholar]

13. Pati S, Chauhan AS, Panda M, Swain S, Hussain MA. Neonatal care practices in a tribal community of Odisha, India: a cultural perspective. J Trop Pediatr. 2014 Jun 1;60(3):238-44. [PubMed] [Google Scholar]

14. Shah S, Sharma G, Chandra T, Chandra R, Sapkota NK. Newborn care practices in a Jhangad community of Eastern Nepal. Int J Health Sci Res. 2015 Dec;5(12):613. [Google Scholar]

15. Mersha A, Assefa N, Teji K, Shibiru S, Darghawth R, Bante A. Essential newborn care practice and its predictors among mother who delivered within the past six months in Chencha District, Southern Ethiopia, 2017. PLoS One. 2018 Dec 11;13(12):e0208984. [PubMed] [Google Scholar]

16. Bahurupi YA, Acharya S, Shinde R. Perceptions and practices of traditional birth attendants in a tribal area of Maharashtra: A qualitative study. The Health Agenda. 2013;1(3):77-83. [Google Scholar]

17. Gedamu H, Tsegaw A, Debebe E. The prevalence of traditional malpractice during pregnancy, child birth, and postnatal period among women of childbearing age in Meshenti Town, 2016. Int J Reprod Med. 2018;2018: 5945060. [PubMed] [Google Scholar]

18. Chand SB. Cultural beliefs and traditional rituals about child birth practice in Rural, Nepal. MOJ Public Health. 2016 Nov 3;4(7):00106. 19. 
19. Kumar V, Shearer JC, Kumar A, Darmstadt GL. Neonatal hypothermia in low resource settings: a review. J Perinatol. 2009 Jun;29(6):401-12. [PubMed] [Google Scholar]

20. Reshma, Sujatha R. Cultural practices and beliefs on newborn care among mothers in a selected hospital of Mangalore Taluk. Nitte Univ J Health Sci. 2014 Jun;4(2):21-6. [Google Scholar]

21. Karahan N, Aydın R, Güven DY, Benli AR, Kalkan NB. Traditional health practices concerning pregnancy, birth, and the postpartum period of women giving birth in the hospital. South Clin Ist Euras. 2017;28(3):190-8. [Google Scholar]

22. Saxena V, Jelly P, Sharma R. An exploratory study on traditional practices of families during the perinatal period among traditional birth attendants in Uttarakhand. J Family Med Prim Care. 2020;9(1):15661. [PubMed] [Google Scholar]

23. Coffey PS, Brown SC. Umbilical cord-care practices in low- and middle-income countries: a systematic review. BMC Pregnancy Childbirth. 2017 Dec;17(1):68. [PubMed] [Google Scholar]

24. Webindia123 [Internet]. Religious Practices Sacraments among Hindus ; 2021 [cited 2021 Sep 19]. Available from: https://www.webindia123.com/ goa/people/sacraments.htm

25. Morris JL, Short S, Robson L, Andriatsihosena MS. Maternal health practices, beliefs and traditions in Southeast Madagascar. Afr J Reprod Health. 2014;18(3):101-17. [PubMed] [Google Scholar]

26. Nutrition Care of Rochester [Internet]. Learning from India's Postpartum Traditions; [cited 2021 Sep 19]. Available from: https://www.nutritioncareofrochester. com/article.cfm?ArticleNumber $=30$

27. Sasikala P, Conjeevaram J, Chandrasekhar V, Kumar C, Bhaskar S. A study on traditional beliefs and practices in new-born care among women attending UHTC, NMC, Nellore, A.P. Nat J Res Comm Med. 2017 Jun 30;6(2):120. [Google Scholar]

28. Bangari A, Thapliyal SK, Ruchi R, Aggarwal B, Sharma $U$. Traditional beliefs and practices in newborn care among mothers in a tertiary care centre in Dehradun, Uttarakhand, India. Int J Community Med Public Health. 2019 May 27;6(6):2600-4. [Google Scholar]

29. Webindia123 [Internet]. Religious practices Sacraments among christians; 2021 [cited 2021 Sep 19]. Available from: https://www.webindia123.com/ GOA/PEOPLE/sacraments1.htm

30. Webindia123 [Internet]. Religious practices Sacraments among muslims; 2021 [cited 2021 Sep 19]. Available from: https://www.webindia123.com/ goa/people/muslim.htm

31. Udgiri R. Cultural practices related to postnatal care: a hospital-based study. J Sci Soc. 2017;44(3):152-5. [Google Scholar]

32. Turner C, Pol S, Suon K, Neou L, Day NP, Parker M, Kingori P. Beliefs and practices during pregnancy, post-partum and in the first days of an infant's life in rural Cambodia. BMC Pregnancy Childbirth. 2017 Dec;17(1):116. [PubMed] [Google Scholar]

33. Sunanda B, Paul S. A study on the cultural practices of postnatal mothers in selected hospitals at Mangalore. Nitte Univ J Health Sci. 2013 Sep;3(3):48-53. [Google Scholar] 


\section{Annexure \\ Questionnaire}

\section{Part I: Socio-Demographic Details}

Name:

Age:

Parity:

Religion: (a) Hindu (b) Catholic (c) Muslim

Residence: (a) Rural (b) Urban/ (a) Local (b) Migrant

Occupation:

Education:

Type of family: (a) Nuclear (b) Joint (c) Three generation

Size of family: members

Total monthly income of working members of the family: INR

\section{Part II: Newborn Care Practices}

1. How many hours after delivery was breastfeeding initiated?
(a) Within 30 mins
(b) Within 60 mins
(c) Within 24 hours
(d) Within 48 hours
(e) After 48 hours

2. Do you feel colostrum should be given to the baby?
(a) Yes
(b) No
(c) If no, why?

3. What type of clothes did you use to wrap the newborn baby?
(a) Old washed clothes
(b) New washed clothes
(c) New unwashed clothes

4. What was the timing of the first bath of the baby?
(a) Day 1
(b) Day 2
(c) Day 3
(d) Day 4
(e) Day 5
(f) Later, then specify

5. Did you apply any dressing to the umbilical stump of the baby?
(a) Yes
(b) No

- If yes, what?
(a) Oil
(b) Turmeric
(c) Antiseptic
(d) Other 
6. Was there a desire for the burial of placenta but was not possible?
(a) Yes
(b) No

\section{Part III: Postnatal Care Practices}

1. Did you give pre-lacteal foods to the baby before initiation of exclusive breastfeeding?
(a) Yes
(b) No
(c) If yes, which foods?

2. Were you advised to give formula feeds to your newborn baby?
(a) Yes
(b) No
(c) If yes, for what reason?

3. How was the baby's cord handled once it fell off?
(a) Burial
(b) Hid the cord in their home
(c) Kept cord in amulets
(d) Gave cord to an infertile couple
(e) Any other, then specify

4. Did you use protective amulets for your baby to ward off the evil eye?
(a) Yes
(b) No

- If yes, what in particular did you use?
(a) Bangle
(b) Chain
(c) Anklet
(d) Holy thread
(e) Kajal

5. Did you follow any of the belowmentioned practices related to hygiene in the immediate postnatal period?
(a) Applying turmeric and mango pulp paste to the body while having bath
(b) Body massage with oil before having bath
(c) Splashing hot water/ giving steam to episiotomy wound for faster wound healing
(d) Any other, then specify

6. Do you believe in giving massage to your baby?
(a) Yes
(b) No

- If yes, when was the massage given to the baby?
(a) Before bath
(b) After bath

- How frequently was the massage given?
(a) Daily
(b) Weekly
(c) Monthly

- At what time of the day was the massage given?
(a) Morning
(b) Evening 
- Who gave massage to the baby?
(a) Mother
(b) Other family member
(c) Massage specialist

- What medium was used for massage?
(a) Commercial baby oil
(b) Coconut oil
(c) Baby lotion
(d) Astringent
(e) Any other, then specify

7. Did you follow any of the traditional practices mentioned below post-delivery?
(a) Observing 'Sotti' (staying in hospital till day 6 after delivery)
(b) Purification ceremony of mother and baby
(c) Naming ceremony of the baby
(d) Keeping knife/ broomstick below the bed of mother and cradle of baby
(e) Exposing baby to dhoop after bath
(f) First hair cutting ceremony of baby
(g) Avoiding visitors to ward off the evil eye
(h) Confinement of mother and baby at home for $\mathbf{4 0}$ days
(i) Any other, then specify 